\title{
Bitácora electrónica de obra pública: entre el gobierno electrónico y el abierto
}

\author{
Araceli Pinacho Ríos, Christian Arturo Cruz y Oscar David Valencia
}

\begin{abstract}
Resumen
Aunque el gobierno electrónico y el gobierno abierto son diferentes, hay una relación entre ambos, gracias a los aportes tecnológicos, en objetivos como la transparencia y la rendición de cuentas. Por otra parte, la Bitácora Electrónica de Seguimiento a Obra Pública (BESOP) es una herramienta tecnológica que contribuye precisamente a cumplir esas metas. Sin embargo, no ha tenido la difusión ni el reconocimiento como una herramienta de gobierno abierto. Este ensayo busca su divulgación, para su estudio y fortalecimiento como un medio que ofrece el gobierno electrónico para la consolidación de un gobierno abierto.
\end{abstract}

Palabras clave: gobierno electrónico, gobierno abierto, bitácora de obra pública.

\section{ELECTRONIC LOGBOOK OF PUBLIC WORKS: BETWEEN ELECTRONIC AND OPEN GOVERNMENT}

\begin{abstract}
Despite the differences between the electronic government and the open government, there is a relation between both, thanks to the contributions of technology, in objectives such as transparency and accountability. Precisely, the Electronic Public Works Booklog (BESOP) is a technological tool that contributes to such goals. However, this logbook has not had the proper recognition as an open government tool. This paper seeks to make it known, for its study and strengthening as a way, offered by the electronic government, for the consolidation of the open government.
\end{abstract}

Keywords: electronic government, open government, accountability and transparency in information.

Dol: http://doi.org/10.22201/codeic.16076079e.2020.v21n3.a4 


\section{Araceli Pinacho Ríos}

chely.pinacho17@gmail.com

https://orcid.org/0000-0003-3220-0552

Licenciada en Administración de Empresas Turísticas por la Universidad del Golfo de México (campus Oaxaca), Maestra en Gobierno Electrónico por la Universidad de la Sierra Sur (Unsıs). Se desempeñó en áreas administrativas de la dependencia Caminos y Aeropistas de Oaxaca.

\section{Christian Arturo Cruz}

acm christian@yahoo.com.mx https://orcid.org/0000-0002-6105-9167

Doctor en Ciencias Políticas y Sociales, con orientación en Administración Pública, por la unAM, Catedrático CONACYT, Comisionado a la División de Posgrado de la Universidad de la Sierra Sur, Miembro del Sistema Nacional de Investigadores nivel Candidato.

\section{Oscar David Valencia}

institutointernacionaldeinvest@gmail.com https://orcid.org/0000-0002-2794-1703

Profesor investigador de la Universidad de la Sierra Sur y Director del Instituto de Estudios Municipales de la misma. Doctor en Economía Política del Desarrollo por el Centro de Estudios del Desarrollo Económico y Social de la Benemérita Universidad Autónoma de Puebla. 


\section{Introducción}

Hay diversas cuestiones que surgen ante la problemática presentada en México y gran parte de América Latina sobre el tema de transparencia y acceso a la información. En ese sentido, se reportó, en 2020, que México se encuentra en el lugar 130 de 180 países en el Índice de Percepción de la Corrupción elaborado por Transparencia Internacional (Transparencia Mexicana, 2020).

Lo anterior impulsó la creación de la Nueva Gestión Pública con el objetivo de promover la eficiencia en el uso y la distribución de los recursos públicos y mejorar la calidad de los servicios prestados, entendiendo la tecnología como una herramienta, lo que dio origen al gobierno electrónico. Otro resultado que buscaba esta reforma son las políticas para generar mayor transparencia y acceso a la información.

El gobierno electrónico y el gobierno abierto son diferentes, pero se les vincula por las ventajas que ofrece el primero al segundo. Con el fin de realizar un análisis de esta relación, se consideró la BESOP como una herramienta tecnológica que contribuye al gobierno abierto y a sus pilares de trasparencia, rendición de cuentas, participación y colaboración. A su vez, la Besop cuenta como un medio para lograr un control de la obra pública, así como la transparencia en general, y la rendición de cuentas.

\section{Las reformas a la administración pública}

El sector gubernamental ha realizado tareas en relación a la satisfacción de las necesidades de los ciudadanos para hacer más eficientes los procesos administrativos a través de la descentralización de funciones. Por esto, se ha establecido el uso de las Tecnologías de la Información y Comunicación (тіc). Sin embargo, en los últimos años, las organizaciones públicas siguen presentado diversos problemas, uno de ellos es la incapacidad de resolver eficiente y eficazmente las actividades que tiene a su cargo, aunque cuenten con las herramientas tecnológicas adecuadas.

Así, la Nueva Gestión Pública surge bajo la necesidad de transformar la administración pública y la eliminación de la burocracia. También busca la menor intervención estatal y fomentar una ciudadanía con democracia plena, para que no se vea al aparato gubernamental como un dictador de la ley al que no se debe cuestionar por el cumplimiento de sus obligaciones. Como enfatiza Aguilar (2006) en su definición sobre la Nueva Gestión Pública:

El cambio de las normas, las estructuras organizacionales y los patrones directivos y operativos del gobierno, que por sus propósitos, modos o resultados se orienta a formas posburocráticas de organización, dirección y operación, con el fin de elevar la eficiencia, la calidad y la responsabilidad de la acción pública (p. 146). 
Es decir, resulta necesario dejar de lado la administración tradicional y procesos burocráticos para dar paso a una reforma posburocrática; y con ello generar un cambio en el esquema tradicional, así como la reducción de costos en la administración y mayor eficiencia en los servicios.

Para lograr la eficiencia en las actividades administrativas, el gobierno se ve en la necesidad de hacer uso de las tecnologías, en conciencia de que la ciudadanía cada día se encuentra en crecimiento y cada vez es más difícil, por no decir imposible, satisfacer las necesidades o cubrir los servicios demandados sin caer en la burocracia. Aunado al cambio en los procesos de la administración y con el apoyo en tecnologías es como se presenta el surgimiento del gobierno electrónico.

\section{El gobierno electrónico}

Surge en la década de los noventa cuando se comenzó a plantear que el uso de las nuevas tecnologías permitía la transformación de las actividades gubernamentales. Se consideró internet como un medio de comunicación bidireccional, para la realización de negocios, de diversión y educativo, para adquirir conocimientos, entre muchas otras labores.

Así inició el gobierno electrónico, cuyo principal antecedente mundial en cuestión de iniciativa de mejora y reforma gubernamental fue durante la presidencia de Bill Clinton. A partir de esta administración, se consideró el modelo para su implementación en países desarrollados, los cuales pretendían un avance hacia la sociedad de la información y el conocimiento. Desde dichas iniciativas y consecuencia del uso de las TIc, sobre todo de internet en los procesos del sector gubernamental, surge el concepto de gobierno electrónico (Ruedas y Peréz, 2006, p.1).

A continuación, presentaré algunas definiciones para tener un panorama más amplio del concepto de gobierno electrónico. La Secretaría de la Función Pública (2012) lo refiere como un aprovechamiento de las tıc en la mejora de la gestión interna de la administración pública, para otorgar mejores servicios, facilitar el acceso a la información, rendición de cuentas, transparencia y fortalecer la participación ciudadana. Esta definición se enfoca en la relación del gobierno con los diversos sectores como la industria, la ciudadanía y el mismo gobierno.

\section{Gobierno abierto}

Nace en 2009, a raíz del memorando del presidente estadounidense Barack Obama, el "Memorándum de Transparencia y Gobierno Abierto", donde se comprometía a tener un gobierno basado en la transparencia, la participación y 
la colaboración. Años después se le agregaría la rendición de cuentas, surgiendo como resultado una nueva tendencia en la administración pública a la que intentar conceptualizar. Algunas de estas definiciones hablan del uso de las Tic en el sector público con el propósito de posibilitar la transparencia y entrega de cuentas; así, la ciudadanía puede conocer las prioridades y objetivos del gobierno y éste adquirir mayor responsabilidad en el uso de los recursos públicos (Vargas, 2011). Con base en esto y en pretender legitimar al gobierno ante la ciudadanía para abatir la desconfianza en el mismo, surge el gobierno abierto que se define como:

[...] un gobierno que abre sus puertas al mundo, co-innova con todos, especialmente con los ciudadanos; comparte recursos que anteriormente estaban celosamente guardados, y aprovecha el poder de la colaboración masiva, la transparencia en todas sus operaciones, y no se comporta como un departamento o una jurisdicción aislada, sino como una estructura nueva, como una organización verdaderamente integrada y que trabaja en red (Don, 2010,

Es précis... resaltar que el gobierno abierto es un nuevo paradigma, y que para su cumplimiento se basa en la transparencia, rendición de cuentas, participación ciudadana y colaboración, lo que permite el cambio de la relación entre la ciudadanía y el Estado. Propone trabajar colaborativamente sobre las debilidades y fortalezas del gobierno para que la ciudadanía o los mismos gobiernos propongan soluciones y estén involucrados. Para este caso, la transparencia en la información y rendición de cuentas son los pilares que pueden coadyuvar en el reconocimiento de la BESOP como herramienta de gobierno abierto.

Cada día se presenta mayor exigencia para que la información sea más abierta, completa y oportuna. No sólo es necesario tener a disposición la información requerida, sino que debe ser de fácil acceso para el público y que los datos puedan estar de manera accesible a través de tecnologías de la información y la comunicación (Naser y Ramírez, 2014).

Sin embargo, el gobierno aún no alcanza la consolidación de un gobierno abierto, pues no ha logrado cumplir en materia de transparencia y rendición de cuentas, según las expectativas de la sociedad, quien debe tomar un papel más exigente y valorar como derecho el fácil y libre acceso a la información pública. De acuerdo con lo anterior, la Carta Iberoamericana de Gobierno Abierto menciona que:

La transparencia implicará dos ámbitos cruciales: el derecho de acceso a la información que está en poder de las instituciones públicas y que puedan solicitar los ciudadanos, y también la obligación de los gobiernos de poner a disposición de la ciudadanía, de forma proactiva, aquella información que den cuenta de sus actividades, del uso de los recursos públicos y sus resultados, con base en los principios de la publicidad activa, tales como la relevancia, exigibilidad, accesibilidad, oportunidad, veracidad, comprensibilidad, sencillez y máxima divulgación (Centro Latinoamericano de Administración para el Desarrollo, 2016, p. 4).

Esto hace referencia al segundo pilar del gobierno abierto sobre la responsabilidad de los servidores públicos, y del mismo gobierno a dar respuesta a las peticiones 
de la sociedad civil, al igual que al cumplimiento de las autoridades. De aquí surge la rendición de cuentas que se define por la Carta Iberoamericana del Gobierno Abierto como un

[...] pilar [que] se basa en las normas, procedimientos y mecanismos institucionales como deber legal y ético que obligan a las autoridades gubernamentales a fundamentar sus acciones y asumir la responsabilidad sobre sus decisiones, responder oportunamente e informar por el manejo y los rendimientos de fondos, bienes o recursos públicos asignados, y por los resultados obtenidos en el cumplimiento del mandato conferido (Centro Latinoamericano de Administración para el Desarrollo, 2016, p.6).

Es importante mencionar que los elementos considerados en la rendición de cuentas son "dos sujetos involucrados, un asunto sobre el que se rinden cuentas, relacionado necesariamente con una responsabilidad asumida por el primer sujeto y un juicio y/o una sanción emitida por el segundo sujeto" (López y Merino, 2009, p. 2).

\section{La BESOP, relación gobierno electrónico y gobierno abierto}

Gobierno electrónico y gobierno abierto son tendencias actuales de la Administración Pública, originadas a fines del siglo xx y principios de xxı. Retoman elementos que en sí mismos no son nuevos (uso de tecnología y los pilares del GA), surgieron de memorandos, y se han convertirdo en objeto de estudio académico y en estrategia de gobierno y modernización administrativa, al grado de llegar a tener compromisos y cartas internacionales. Es cierto que están relacionados por la utilización de TIc, pero no deben de ser confundidos. El gobierno abierto se auxilia de los elementos tıc que aporta el gobierno electrónico, como portales web, aplicaciones móviles, datos abiertos y otros como la Bitácora Electronica de Obra Pública. La imagen 1 muestra la relacion de ambos conceptos.

Imagen 1. Relación gobierno electrónico y gobierno abierto (Cruz, 2016).

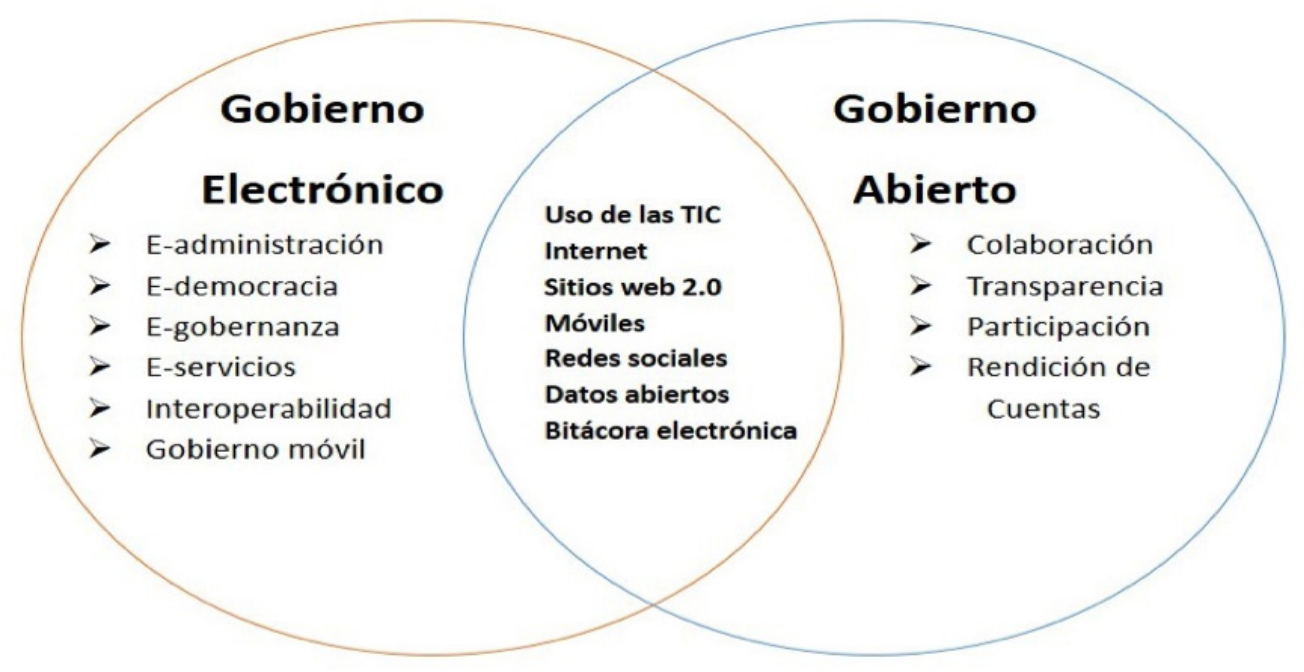


Imagen 2. Bitácora de Obra Pública tradicional. Cortesía de Caminos y Aeropistas de Oaxaca.
Esta herramienta tiene como antecedente haber sido utilizada por Petróleos Mexicanos (PEMEX) y en el 2007 se transfiere a la Secretaría de la Función Pública (SFP), iniciando así una implementación nacional. Se sabe que organismos como la Comisión Nacional del Agua (CONAGUA), CFE, sct y Caminos y Aeropistas de Oaxaca (CAO) han implementado este software con el fin de reportar los avances de obra que se están llevando a cabo y con ello se propicia la transparencia en el uso de los recursos públicos de la federación. Así, la BEsop es definida por la Secretaría de la Función Pública (2011) como:

[...] el Programa Informático Autorizado por la Secretaria de la Función Pública que por medios remotos de comunicación electrónica entre las partes de un contrato de obra pública o de servicios relacionados con la misma, en la que se registran los asuntos y eventos importantes que se presentan durante la ejecución de los trabajos (p. 3).

El gobierno, para el control de la obra pública, se apoyaba en bitácoras tradicionales, las cuales estaban hechas en papel y se llenaban a mano. Éstas tenían como limitantes el error ortográfico, poca legibilidad, daño por condiciones del ambiente y no eran consideradas para aportar en temas de transparencia. La imagen 2 muestra una bitácora tradicional.

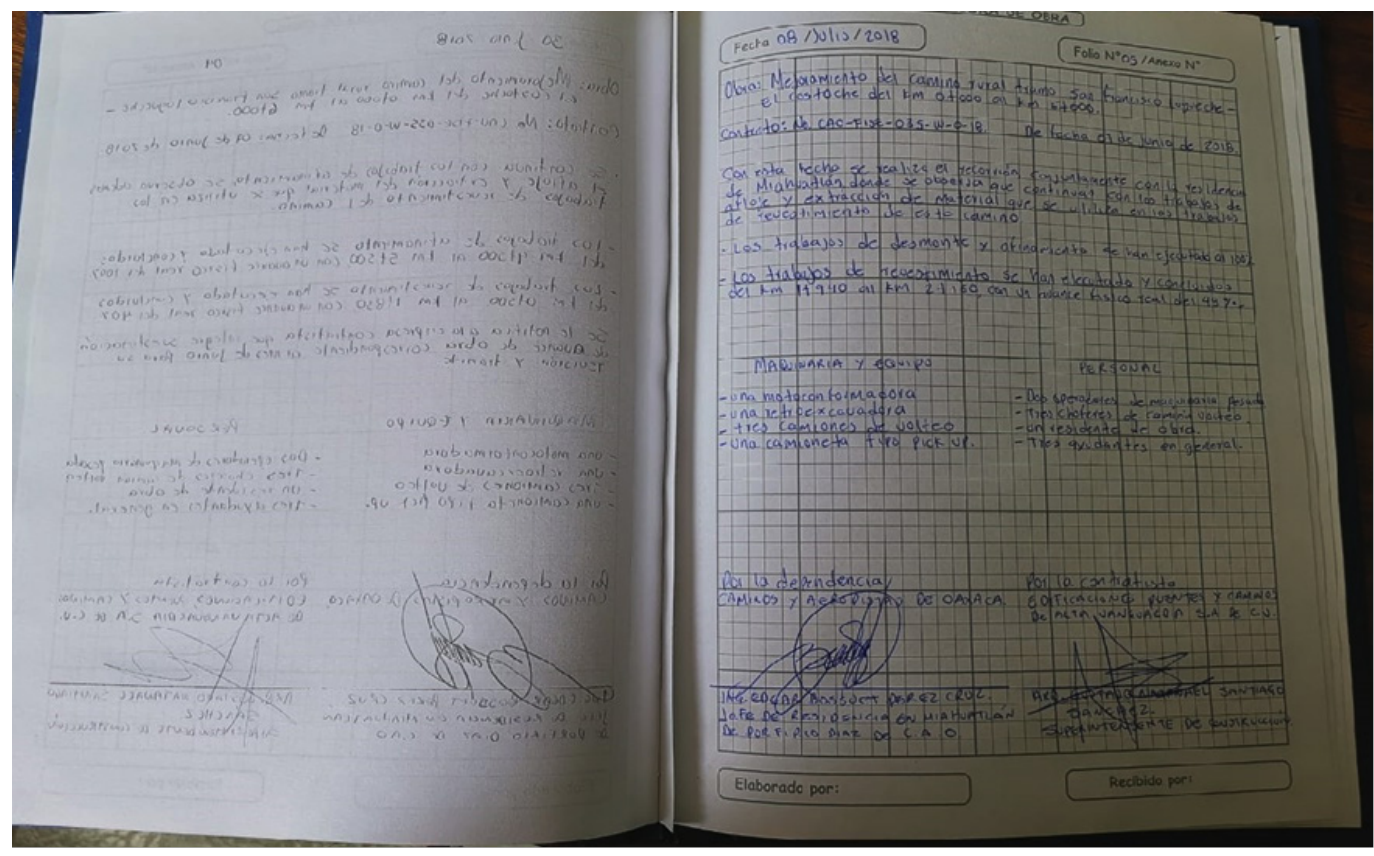

La llegada de la BESop trajo consigo beneficios para el control de obra y gobierno abierto, tales como:

- Información en tiempo real de lo que sucede en una obra.

- Seguridad de manejo de información.

- Implementación de Firma Electrónica Avanzada para garantizar la autenticidad de la información (ver imagen 3).

- Información veraz, oportuna y clasificada. 
Imagen 3. Firmado electrónico de la BEOP. Fuente: Bitácora Electrónica de Seguimiento a la Obra Pública, Guía de Usuario Final.

\section{Firma Electrónica}

Paso 1.- Proporcione los elementos de su firma electrónica.

Lave Privada ("key)

Certificado (r.cen)

Contraseha
Clic aquí para ingresar tus datos

Que las dependencias o entidades puedan respaldar su información de manera digital e impresa.

De esta forma, se está ante una herramienta tecnológica (gobierno electrónico), que contribuye al gobierno abierto, lo que muestra la relación entre ambos conceptos y su complementariedad, a pesar de que mantienen su naturaleza y particularidades.

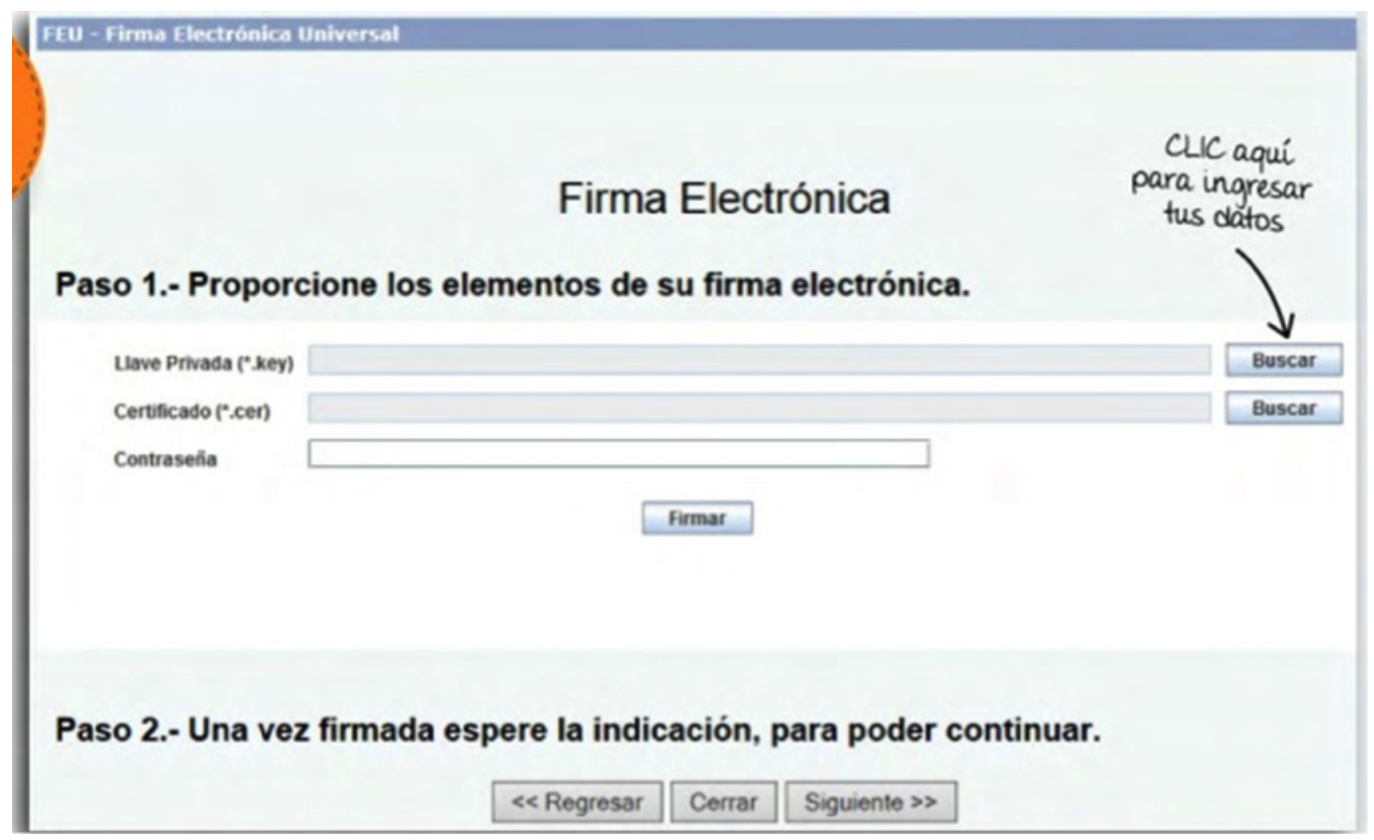

\section{Conclusión}

La BESOP es un ejemplo de la relación entre el gobierno electrónico y gobierno abierto. El uso de la tecnología debe tener un sentido, una razón de ser; en este caso es la transparencia, el acceso a la información y la rendición de cuentas (horizontal), para la consolidación de gobiernos abiertos. La BEsop todavía no es considerada por sectores académicos o gubernamentales como una herramienta de gobierno abierto, pero aquí se argumenta que sí puede ser vista como tal. Además, esperamos que el presente ensayo contribuya a darla a conocer, no sólo como una herramienta administrativa, sino también por sus aportes a la transparencia y la rendición de cuentas.

\section{Referencias}

* Águilar, L. F. (2006). Gestión Pública y Gobernanza. Fondo de Cultura Económica.

* Cámara de Diputados (2015, 4 de mayo). Ley General de Transparencia y Acceso a la Información Pública. http://www.diputados.gob.mx/LeyesBiblio/pdf/LGTAIP.pdf.

* Cámara de Diputados (2016, 9 de mayo). Ley Federal de Transparencia y Acceso a la Información Pública [reforma del 27-01-2017]. http://www.diputados.gob.mx/ LeyesBiblio/pdf/LFTAIP 270117.pdf. 
"Bitácora electrónica de obra pública: entre el gobierno electrónico y el abierto" Araceli Pinacho Ríos, Christian Arturo Cruz y Oscar David Valencia

* Centro Latinoamericano de Administración para el Desarrollo (2016). Carta Iberoamericana de Gobierno Abierto. https://www.sfp.gov.py/sfp/archivos/ documentos/CIGA 2016\%20final knawtdh9.pdf.

* Cruz, Christian. (2016). Gobierno electrónico y gobierno abierto. Estado actual del debate conceptual. Revista de Administración Pública, LI(140), 85-85.

* Don, T. (2010). De Gobierno Abierto a Estado Abierto. https://biblioguias.cepal.org/ EstadoAbierto/concepto.

* Fountain, J. (2001). La Construcción del Estado Virtual: Tecnologias de Información y cambio institicional. CIDE.

* López Ayllón, S. y Merino, M. (2009). La rendición de cuentas en México: perspectivas y retos. https://archivos.juridicas.unam.mx/www/bjv/libros/6/2800/4.pdf.

* Naser, A., y Ramírez, A. (2014). Plan de Gobierno Abierto. Una hoja de ruta para los gobiernos dela región. cEPAL. https://www.cepal.org/ilpes/noticias/paginas/3/54303/ Plan de Gobierno Abierto.pdf.

* Ruedas, A., y Peréz, P. (2006). El Gobierno Electrónico: su estudio y perspectivas de desarrollo. Razón y palabra, 52. http://www.razonypalabra.org.mx/anteriores/ n52/27RuelasyAramburo.pdf.

* Secretaría de la Función Pública. (2011). Bitácora Electrónica de Obra Pública. Manual del Usuario para el Administrador de Dependencia y Entidad Federativa. https://www. uv.mx/contraloria/files/2013/02/18.Manual-de-Usuario-para-el-Administradorde-Dependencia-y-entidad-Federativa.pdf.

* Secretaría de la Función Pública. (2012). Gobierno Digital. http://2006-2012. funcionpublica.gob.mx/index.php/unidades-administrativas/ssfp/mejorgobierno/faq/gobierno-digital.html.

* Transparencia Mexicana. (2020). México detiene caída en el Índice de Percepción de la Corrupción: Transparencia Mexicana. Obtenido de https://www.tm.org.mx/ipc2019/

* Presidencia del Consejo de Ministros y Oficina Nacional de Gobierno Electrónico e Informatica. (2013). Una mirada al Gobierno Electrónico en el Perú. La oportunidad de acercar el Estado a los ciudadanos a través de las Tic. http://Www2.congreso.gob.pe/ sicr/cendocbib/con4 uibd.nsf/OD6D8CA5D781070305257E9200775428/\$FILE/3 pdfsam libro ongei.pdf.

* Vargas, C. (2011). El Gobierno Electrónico o e- Gobierno. Uni-pluri/versidad, 11(1), s.p. http://www2.congreso.gob.pe/sicr/cendocbib/con4 uibd.nsf/BD9EBOAFF7944 2F705257C170009C981/\$FILE/9711.pdf.

\section{Cómo citar el artículo}

* Pinacho Ríos, Araceli, Cruz, Christian Arturo y Valencia, Oscar David. (2020). Bitácora electrónica de obra pública: entre el gobierno electrónico y el abierto. Revista Digital Universitaria (RDU) Vol. 21, núm. 3 mayo-junio. Dol: http://doi. org/10.22201/codeic.16076079e.2020.v21n3.a4. 